\title{
ALIMENTAÇÃO E COMPORTAMENTO DE LARVAS DE PACU, Piaractus mesopotamicus (Holmberg, 1887)
}

\author{
Feeding and behavior of pacu, Piaractus mesopotamicus (Holmberg, 1887) larvae
}

\author{
Eduardo Lopes Beerli ${ }^{1}$, Priscila Vieira Rosa Logato ${ }^{2}$, Rilke Tadeu Fonseca de Freitas ${ }^{3}$
}

\begin{abstract}
RESUMO
Conduziu-se este trabalho com o objetivo de avaliar o efeito da utilização de dietas naturais e artificiais sobre o desempenho e comportamento de larvas de pacu (Piaractus mesopotamicus), entre o $2^{\circ}$ e o $10^{\circ}$ dia de vida. Foram utilizadas 30 caixas plásticas, cada uma com 30 litros de água e renovação contínua, onde as larvas foram mantidas durante o período experimental. Cada caixa recebeu 10 larvas por litro, totalizando 300 larvas/caixa. Foram testados 6 tratamentos, cada qual com 5 repetições. Os tratamentos foram: T1-ração, T2-plâncton, T3-artêmia, T4-plâncton + ração, T5-artêmia + ração e T6-artêmia + plâncton. As larvas foram alimentadas 6 vezes ao dia, nos horários de 4, 8, 12, 16, 20 e 24 horas. A temperatura da água foi mantida constante a $27^{\circ} \mathrm{C}$, o oxigênio dissolvido permaneceu na faixa de $6,16 \pm 0,34$ e o pH, entre 7,16 $\pm 0,22$. Aos $2,4,6,8$ e 10 dias de vida, foram coletadas amostras de 30 larvas para determinação do comprimento total e peso. No final do experimento ( $10^{\circ}$ dia), as larvas que receberam artêmia + plâncton (T6) alcançaram os maiores valores de comprimento total $(8,35 \mathrm{~mm})$ e peso corporal $(3518 \mu \mathrm{g})$, em relação a todas as outras dietas testadas. As larvas devem permanecer em laboratório por um período de 6 dias após a eclosão, recebendo alimento do terceiro ao sexto dia. A partir do sexto dia, as larvas estão com a vesícula gasosa completamente inflada e apresentam nado contínuo.
\end{abstract}

Termos para indexação: Peixes, larvicultura, piscicultura, dieta.

\begin{abstract}
The objective of this research was to evaluate the effect of natural and artificial diets on the development and behavior of pacu (Piaractus mesopotamicus) larvae, between the $2^{\text {nd }}$ and $10^{\text {th }}$ day post hatch. The larvaes were kept in $30 \mathrm{~L}$ - plastic boxes ( $\mathrm{n}=30$ boxes), in constant flow-through. The larvae density was 10 post-larvae/L (300 larvae/box). Six diets with 4 sampling days $(4,6,8$ and 10 days post-hatch, $\mathrm{n}=30$ larvae) using 5 repetitions were tested. The diets were: T1- commercial feed, T2plancton, T3- artemia, T4- plancton + feed, T5- artemia + feed and T6- artemia + plancton. Larvaes were fed 6 times a day: 4, $8,12,16,20$ and 24 hours. The water temperature was kept constant at $27^{\circ} \mathrm{C}$, dissolved oxigen was kept between $6.16 \pm 0.34$ and $\mathrm{pH}$ between $7,16 \pm 0,22$. At the end of the experiment $\left(10^{\text {th }}\right.$ day post-hatch $)$, the larvaes that received diet containing artemia + plancton (T6) were longer $(8.35 \mathrm{~mm})$ and heavier ( $3518 \mu \mathrm{g}$ body weight), compared to the larvae fed with the other tested diets. The larvae should be in the laboratory for 6 days after hatch, being feed from the $3^{\text {rd }}$ day after hatch. Before the $6^{\text {th }}$ day, the larvae have the gas bladder completely full and swim continuously.
\end{abstract}

Index terms: Fish, larviculture, aquaculture, diet.

(Recebido para publicação em 31 de outubro de 2002 e aprovado em 6 de janeiro de 2003)

\section{INTRODUÇÃO}

O Brasil possui grande potencial hídrico e climático, o que possibilita o cultivo de diversas espécies de peixes. Contudo, a piscicultura ainda apresenta resultados modestos de desenvolvimento, devido aos processos de produção adotados e à falta de informações sobre as espécies nativas com potencial zootécnico.

Mesmo com o aprimoramento das técnicas de reprodução, alimentação e manejo na piscicultura, mui tos problemas precisam ainda ser resolvidos, principalmente com relação à larvicultura de peixes nativos. Diversos fatores interferem na sobrevivência das larvas de peixes, tornando a larvicultura um forte ponto de estrangulamento na produção de grandes quantidades de alevinos.

Nesse contexto, pesquisadores passaram a realizar pesquisas com espécies nativas em laboratório, visando a conhecer dietas eficientes para os primeiros dias de vida, buscando a maximização da produção por

1. Mestrando em Aquacultura. Rua Marechal Rondom, Quadra 20, Lote 2, Casa 2, Setor Tecnorte - 77813-250 - Araguaína, TO.

2. Doutorado em Nutrição de Monogástricos, Departamento de Zootecnia - Universidade Federal de Lavras/UFLA, Caixa Postal 37 - $37200-000$ Lavras, MG.

3. Doutorado em Melhoramento Genético, Departamento de Zootecnia/UFLA. 
meio do fornecimento de condições adequadas e controladas.

Como conseqüência, organismos vivos selvagens, organismos produzidos em laboratório e alimentos inertes vêm sendo utilizados como alimento inicial de larvas.

Objetivou-se com este trabalho avaliar o efeito da utilização de dietas naturais e artificiais sobre o desempenho e comportamento de larvas de pacu (Piaractus mesopotamicus), entre o segundo e o décimo dia de vida.

\section{MATERIAL E MÉTODOS}

O experimento foi realizado no Laboratório de Piscicultura da Estação Ambiental de Itutinga - Cemig (Companhia Energética de Minas Gerais), em Itutinga, MG, no período de 27 de janeiro a 4 de fevereiro de 2001.

Foram utilizadas 30 caixas plásticas de $42 \mathrm{~cm}$ x $63 \mathrm{~cm}$ x $19 \mathrm{~cm}$ de altura, cada uma com 30 litros de água com renovação contínua, onde as larvas foram mantidas durante o período experimental, de 9 dias. $\mathrm{O}$ escoamento da água foi feito com um sistema do tipo "cotovelo de PVC" e se situou do lado oposto ao abastecimento. Do lado interno do cano de escoamento, foi instalada uma tela de malha fina, visando a evitar a fuga das larvas. As laterais das caixas foram pintadas externamente de preto para diminuir o estresse dos peixes. A temperatura foi mantida a $27^{\circ} \mathrm{C}$, utilizando-se um termostato com aquecedor na caixa d'água, que serviu de reserva de água para o experimento.

A eclosão ocorreu no dia 25 de janeiro pela manhã, em incubadoras cilíndricas de 100 litros, onde as larvas permaneceram por dois dias até iniciarem a alimentação exógena. Quando as larvas tinham dois dias de idade, ou seja, no dia 27 de janeiro, foi iniciado o experimento.

Foram medidos o comprimento total de cada larva amostrada utilizando-se paquímetro com precisão de 0,02 mm. Devido ao reduzido peso inicial, as larvas foram pesadas em conjuntos de 30 larvas em balança analítica digital com precisão de $0,001 \mathrm{~g}$ e o peso corporal médio foi calculado em seguida.

Cada caixa recebeu apenas 10 larvas por litro, totalizando 300 larvas/caixa. Essa baixa densidade foi utilizada visando a fornecer aos peixes boas condições para seu desenvolvimento, sem ocorrência de competição pelo alimento ou interferência na qualidade da água. Os tratamentos foram os seguintes: $\mathrm{R}=100 \%$ ração; $\mathrm{P}=100 \%$ plâncton; $\mathrm{A}=100 \%$ artêmia; $\mathrm{R}+\mathrm{P}=50 \%$ ração $+50 \%$ plâncton; $\mathrm{R}+\mathrm{A}=50 \%$ ração $+50 \%$ artêmia e $\mathrm{A}+\mathrm{P}=50 \%$ artêmia $+50 \%$ plâncton.

Foi utilizada ração comercial extrusada com $48 \%$ de proteína bruta, triturada e peneirada, para reduzir as partículas ao tamanho de $75 \mu \mathrm{m}$ ou menos, para que pudessem ser consumidas pelas larvas. A cada 4 horas a ração foi fornecida uniformemente por toda a superfície da água, à vontade.

O plâncton foi coletado diariamente com rede de plâncton de $75 \mu \mathrm{m}$ em um tanque previamente adubado com aproximadamente $500 \mathrm{~g} / \mathrm{m}^{2}$ de esterco bovino curtido. O plâncton coletado foi mantido em alta densidade em um balde com aeração constante e, em cada horário de alimentação, foi fornecido um volume de $400 \mathrm{ml}$ para cada caixa do tratamento 2 (somente plâncton) e 200 ml para cada caixa dos tratamentos 4 e 6 .

A artêmia foi obtida a partir da eclosão diária de cistos, conforme a metodologia a seguir: hidratação dos cistos em água doce por cerca de 20 minutos; colocação dos cistos numa incubadora transparente contendo água com $20 \mathrm{~g}$ de sal por litro a $30^{\circ} \mathrm{C}$, com aeração forte e luminosidade constante, fornecida por lâmpadas fluorescentes ao lado das incubadoras. Após cerca de 24 horas que ocorreu a eclosão e, retirando-se a aeração, os náuplios de artêmia foram concentrados no fundo da incubadora e coletados por sifonamento.

As larvas foram alimentadas 6 vezes ao dia, nos horários de 4, 8, 12, 16, 20 e 24 horas. Para que não houvesse competição, cada um dos alimentos foi fornecido de modo que ocorressem sobras até o momento do próximo horário de alimentação.

A quantidade de cada alimento fornecida nos tratamentos com dois itens alimentares $(\mathrm{R}+\mathrm{P}, \mathrm{R}+\mathrm{A}$ e $\mathrm{A}+\mathrm{P})$ foi a metade da quantidade oferecida em cada tratamento com apenas um item alimentar ( $\mathrm{R}, \mathrm{P}$ e $\mathrm{A})$.

Os parâmetros oxigênio dissolvido e temperatura foram monitorados diariamente pela manhã com aparelhos eletrônicos (YSI 55), assim como o pH (Bernauer Aqüicultura).

No início do experimento, quando as larvas tinham dois dias de vida, foi coletada uma amostra de 30 larvas para determinação do comprimento total inicial e peso inicial. Essas 30 larvas iniciais não fizeram parte das 300 larvas colocadas em cada caixa plástica. Depois do início do experimento, aos 4, 6, 8 e 10 dias de vida, foram coletadas outras amostras aleatórias de 30 larvas (10\% da quantidade inicial) em todas as caixas. Desse modo, ao longo do experimento, a densidade em número de larvas dentro das caixas diminuía a cada coleta. Todas as amostras foram 
armazenadas em frascos contendo formol a $10 \%$ e identificadas.

Também foi observado o comportamento das larvas durante todo o período de experimento, registrando dados referentes ao desenvolvimento e comportamento das mesmas, visando a obter informações de interesse para auxiliar no manejo de cultivo inicial do pacu.

$\mathrm{O}$ experimento foi conduzido em um DIC (delineamento inteiramente casualizado) com parcelas subdivididas. Cada parcela foi constituída por seis tratamentos, e as subparcelas, pelas quatro idades das larvas nos momentos das amostragens (4, 6, 8 e 10 dias), com cinco repetições por subparcela; essa composta por uma amostra de 30 larvas.

As análises estatísticas foram realizadas pelo pacote computacional SISVAR, versão 6.12 (FERREIRA, 1998). Foi utilizado o teste de ScottKnott.

\section{RESULTADOS E DISCUSSÃO}

O comprimento total médio das larvas no início do experimento foi de $5,86 \mathrm{~mm}$ e o peso médio individual foi de $630 \mu \mathrm{g}$.

Durante o experimento, as variáveis oxigênio dissolvido e $\mathrm{pH}$ mantiveram-se dentro dos limites considerados adequados para peixes tropicais, variando de $6,16 \mathrm{mg} / 1$ a $6,65 \mathrm{mg} / \mathrm{l}$ e $6,94 \mathrm{mg} / \mathrm{l}$ a 7,38 $\mathrm{mg} / \mathrm{l}$, respectivamente (Tabela 1 ). Dessa forma, a qualidade da água não influenciou os tratamentos. Deve-se considerar que a baixa densidade de larvas utilizadas por caixa, a renovação constante de água e a limpeza freqüente auxiliaram para manter a qualidade da água.

TABELA 1 - Valores médios e desvio-padrão das variáveis limnológicas observadas durante o período de experimento, nos diferentes tratamentos.

\begin{tabular}{ccc}
\hline Tratamentos & $\begin{array}{c}\text { Oxigênio Dissolvido } \\
(\mathbf{m g} / \mathbf{l})\end{array}$ & pH \\
\hline R & $6,16 \pm 0,34$ & $6,94 \pm 0,22$ \\
P & $6,57 \pm 0,42$ & $7,27 \pm 0,09$ \\
A & $6,53 \pm 0,33$ & $7,38 \pm 0,18$ \\
R+P & $6,63 \pm 0,32$ & $7,25 \pm 0,23$ \\
R+A & $6,33 \pm 0,35$ & $7,16 \pm 0,15$ \\
A+P & $6,65 \pm 0,29$ & $7,36 \pm 0,36$ \\
\hline
\end{tabular}

Aos 4, 6, 8 e 10 dias de vida, os comprimentos totais médios das larvas foram medidos e estão apresentados na Tabela 2

TABELA 2 - Comprimentos totais (mm) das larvas de pacu durante o período de experimento.

\begin{tabular}{ccccc}
\hline \multirow{2}{*}{ Tratamentos } & \multicolumn{4}{c}{ Idade (dias) } \\
\cline { 2 - 5 } & $\mathbf{4}$ & $\mathbf{6}$ & $\mathbf{8}$ & $\mathbf{1 0}$ \\
\hline $\mathrm{R}$ & $6,17 \mathrm{~b}$ & $6,21 \mathrm{c}$ & $6,11 \mathrm{f}$ & $6,17 \mathrm{f}$ \\
$\mathrm{P}$ & $6,13 \mathrm{~b}$ & $6,24 \mathrm{c}$ & $6,57 \mathrm{~d}$ & $7,15 \mathrm{~d}$ \\
$\mathrm{~A}$ & $6,15 \mathrm{~b}$ & $6,33 \mathrm{~b}$ & $7,06 \mathrm{~b}$ & $8,17 \mathrm{~b}$ \\
$\mathrm{R}+\mathrm{P}$ & $6,16 \mathrm{~b}$ & $6,21 \mathrm{c}$ & $6,44 \mathrm{e}$ & $6,80 \mathrm{e}$ \\
$\mathrm{R}+\mathrm{A}$ & $6,16 \mathrm{~b}$ & $6,31 \mathrm{~b}$ & $6,88 \mathrm{c}$ & $7,81 \mathrm{c}$ \\
$\mathrm{A}+\mathrm{P}$ & $6,24 \mathrm{a}$ & $6,39 \mathrm{a}$ & $7,25 \mathrm{a}$ & $8,35 \mathrm{a}$ \\
\hline
\end{tabular}

*Médias com letras diferentes na mesma coluna diferem significativamente $(\mathbf{p}<0,01)$ pelo teste de ScottKnott.

No quarto dia de vida, as larvas que receberam a dieta contendo artêmia + plâncton apresentaram comprimentos totais superiores às larvas dos demais tratamentos, que se mantiveram semelhantes.

Aos 6 dias de vida, as larvas que receberam artêmia isoladamente (A) ou associada a outro alimento $(\mathrm{R}+\mathrm{A}$ e $\mathrm{A}+\mathrm{P})$ tiveram crescimento superior às larvas dos outros tratamentos. O crescimento das larvas do tratamento $\mathrm{A}+\mathrm{P}$ foi superior a todos os demais tratamentos.

A partir do oitavo dia em diante, as larvas de cada tratamento cresceram de maneira distinta, sendo os maiores valores de comprimento obtidos pelo tratamento $\mathrm{A}+\mathrm{P}$, seguido de $\mathrm{A}$.

Os pesos médios das larvas a cada dia de coleta (aos 4, 6, 8 e 10 dias de vida) estão na Tabela 3 .

Com 4 dias de vida, as larvas dos tratamentos $\mathrm{R}+\mathrm{P}$ e $\mathrm{R}+\mathrm{A}$ destacaram-se das demais, apresentando pesos corporais superiores. Considerando-se os maiores comprimentos e os maiores pesos corporais das larvas no quarto dia de vida e daí em diante (Tabelas 2 e 3), observa-se que há um comportamento que mostra melhores resultados no tratamento $\mathrm{A}+\mathrm{P}$. Esse comportamento não foi observado quanto ao peso corporal no quarto dia de vida (Tabela 3), tendo o maior resultado sido obtido pelas larvas do tratamento $\mathrm{R}+\mathrm{A}$. Esse efeito 
pode ter sido causado pelo alimento ingerido, que altera o peso, mas não o comprimento das larvas. Portanto, segundo esses dados, a utilização do comprimento como parâmetro para avaliar o desenvolvimento de larvas é mais preciso do que o peso corporal.

TABELA 3 - Pesos médios $(\mu \mathrm{g})$ individuais das larvas de pacu, pesadas em grupos de 30 larvas, durante o período de experimento.

\begin{tabular}{ccccc}
\hline \multirow{2}{*}{ Tratamentos } & \multicolumn{4}{c}{ Idade (dias) } \\
\cline { 2 - 5 } & $\mathbf{4}$ & $\mathbf{6}$ & $\mathbf{8}$ & $\mathbf{1 0}$ \\
\hline $\mathrm{R}$ & $890 \mathrm{c}$ & $871 \mathrm{e}$ & $800 \mathrm{f}$ & $801 \mathrm{f}$ \\
$\mathrm{P}$ & $880 \mathrm{c}$ & $890 \mathrm{~d}$ & $1351 \mathrm{~d}$ & $1840 \mathrm{~d}$ \\
$\mathrm{~A}$ & $880 \mathrm{c}$ & $1069 \mathrm{c}$ & $1749 \mathrm{~b}$ & $3249 \mathrm{~b}$ \\
$\mathrm{R}+\mathrm{P}$ & $900 \mathrm{~b}$ & $890 \mathrm{~d}$ & $1159 \mathrm{e}$ & $1560 \mathrm{e}$ \\
$\mathrm{R}+\mathrm{A}$ & $920 \mathrm{a}$ & $1089 \mathrm{~b}$ & $1589 \mathrm{c}$ & $2578 \mathrm{c}$ \\
$\mathrm{A}+\mathrm{P}$ & $871 \mathrm{c}$ & $1130 \mathrm{a}$ & $1929 \mathrm{a}$ & $3518 \mathrm{a}$ \\
\hline
\end{tabular}

*Médias com letras diferentes na mesma coluna diferem significativamente $(p<0,01)$ pelo teste de ScottKnott.
No sexto dia de vida, as larvas de quase todos os tratamentos tiveram pesos corporais estatisticamente diferentes entre si. As larvas do tratamento $\mathrm{A}+\mathrm{P}$ apresentaram os maiores pesos e permaneceram com pesos superiores até o final do experimento.

Após o sexto dia, as larvas de todos os tratamentos alcançaram pesos corporais distintos entre si.

Como pode ser observado nas Figuras 1 e 2, ambos os parâmetros monitorados seguiram um padrão muito semelhante, embora os valores tenham escalas diferentes.

Do segundo ao quarto dia de vida, tanto em relação ao comprimento total quanto ao peso corporal, embora tenham ocorrido diferenças significativas e alguns tratamentos tenham se destacado, o crescimento foi bastante homogêneo em todos eles. Isso possivelmente ocorreu devido ao vitelo remanescente que as larvas possuíam. Esse fato é comprovado pela desuniformidade do desempenho das larvas nos subseqüentes dias de coleta e está de acordo com Woynarovich e Horvath (1983). Esses autores observaram que quando a larva inicia a busca de alimento externo, o que assegura sua sobrevivência é a presença de $20 \%$ a $30 \%$ da quantidade de vitelo. Também Jomori (1999) reforça essa teoria, esclarecendo que, com 72 horas (três dias), as larvas de pacu estavam com boca e ânus abertos, bexiga natatória em processo de inflação e ainda possuía resquícios de vitelo.

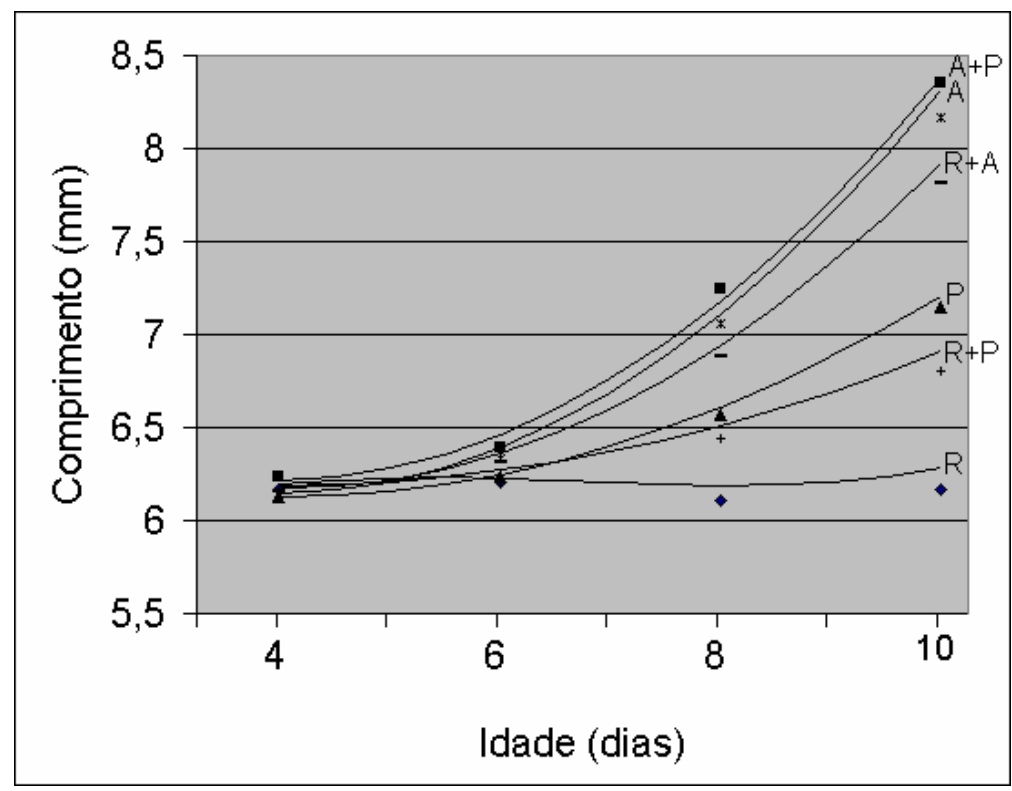

FIGURA 1 - Comprimentos totais (mm) observados e estimados das larvas de pacu durante o período de experimento. $\mathrm{R}=$ ração; $\mathrm{P}=$ plâncton; $\mathrm{A}=$ artêmia; $\mathrm{R}+\mathrm{P}=$ ração+plâncton; $\mathrm{R}+\mathrm{A}=$ ração+artêmia $\mathrm{e}+\mathrm{P}=$ artêmia+plâncton. 
$\mathrm{Na}$ prática, poderia iniciar-se a alimentação por volta do terceiro dia de vida, já que, ao quarto dia, alguns tratamentos desse experimento começaram a se destacar dos demais $(\mathrm{A}+\mathrm{P}$ quanto ao comprimento e $\mathrm{R}+\mathrm{P}$ e $\mathrm{R}+\mathrm{A}$ quanto ao peso corporal), em consequiência da alimentação.

O tratamento constituído unicamente por ração não proporcionou bom desempenho, comparando-se com todos os outros tratamentos (Tabelas 2 e 3 e Figura 3), tanto em relação ao peso quanto ao comprimento. Deve-se considerar que foi utilizada ração comercial extrusada e triturada e essa, quando fornecida, afundava rapidamente, não permanecendo distribuída homogeneamente na coluna d’água. Ainda se devem considerar possíveis perdas de nutrientes por dissolução na água.

As larvas que receberam plâncton apresentaram resultados intermediários entre as que receberam artê- mia e ração (Figuras 3 e Tabelas 2 e 3). O bom desempenho das larvas alimentadas unicamente com plâncton (P) pode ser decorrente dessa composição variada, pois são fornecidos organismos de diversos tamanhos, que nadam em diferentes velocidades, e alguns podem complementar os nutrientes que outros não possuem ou não possuem em quantidades adequadas para as larvas. Deve-se ressaltar que o custo para a produção do plâncton nos viveiros é muito baixo, apenas com a fertilização.

A partir do sexto dia de vida, as larvas que receberam artêmia, tanto individualmente (A) quanto associada a outro alimento $(\mathrm{R}+\mathrm{A}$ e $\mathrm{A}+\mathrm{P})$, destacaram-se das demais, tanto em relação ao comprimento quanto ao peso, mostrando que, entre os itens testados, a artêmia foi um excelente alimento.

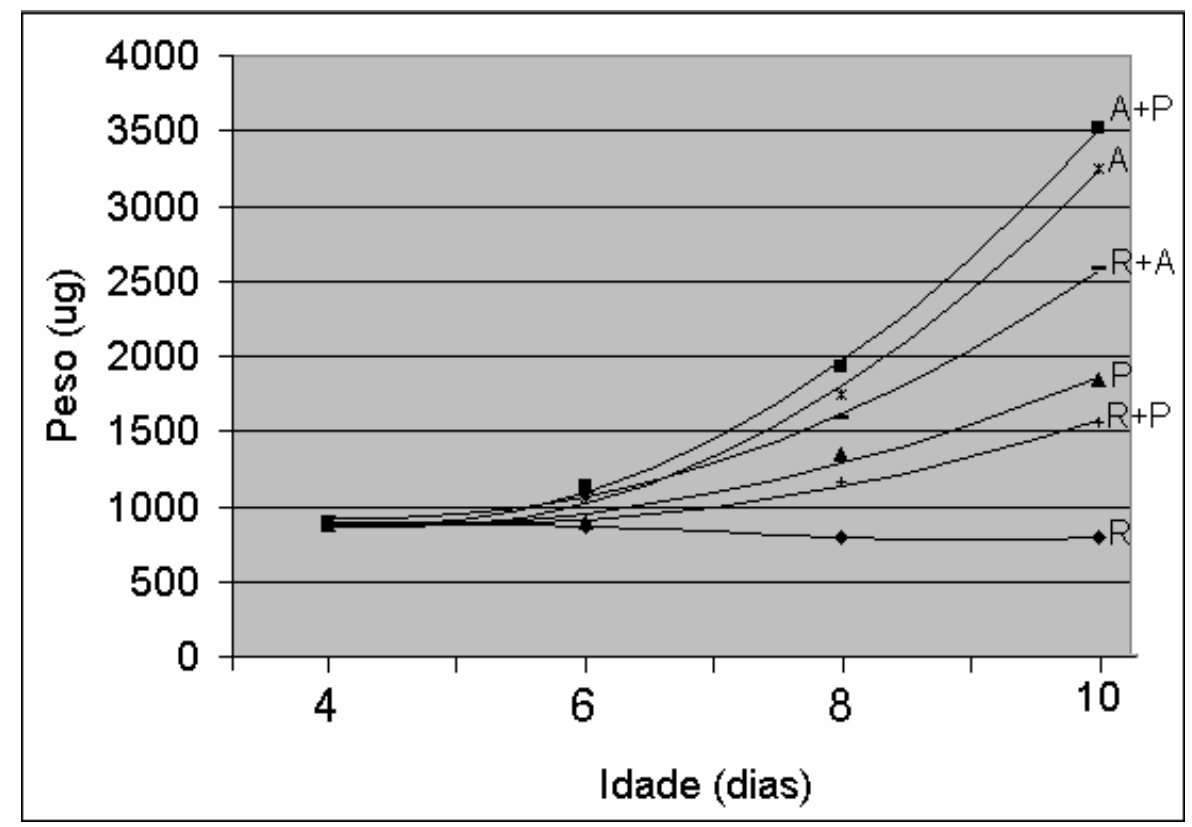

FIGURA 2 - Pesos médios ( $\mu \mathrm{g})$ observados e estimados das larvas de pacu, em grupos de 30 larvas, durante o período de experimento. $\mathrm{R}=$ ração; $\mathrm{P}=$ plâncton; $\mathrm{A}=$ artêmia; $\mathrm{R}+\mathrm{P}=$ ração+plâncton; $\mathrm{R}+\mathrm{A}=$ ração+artêmia e $\mathrm{A}+\mathrm{P}=$ artêmia+plâncton. 


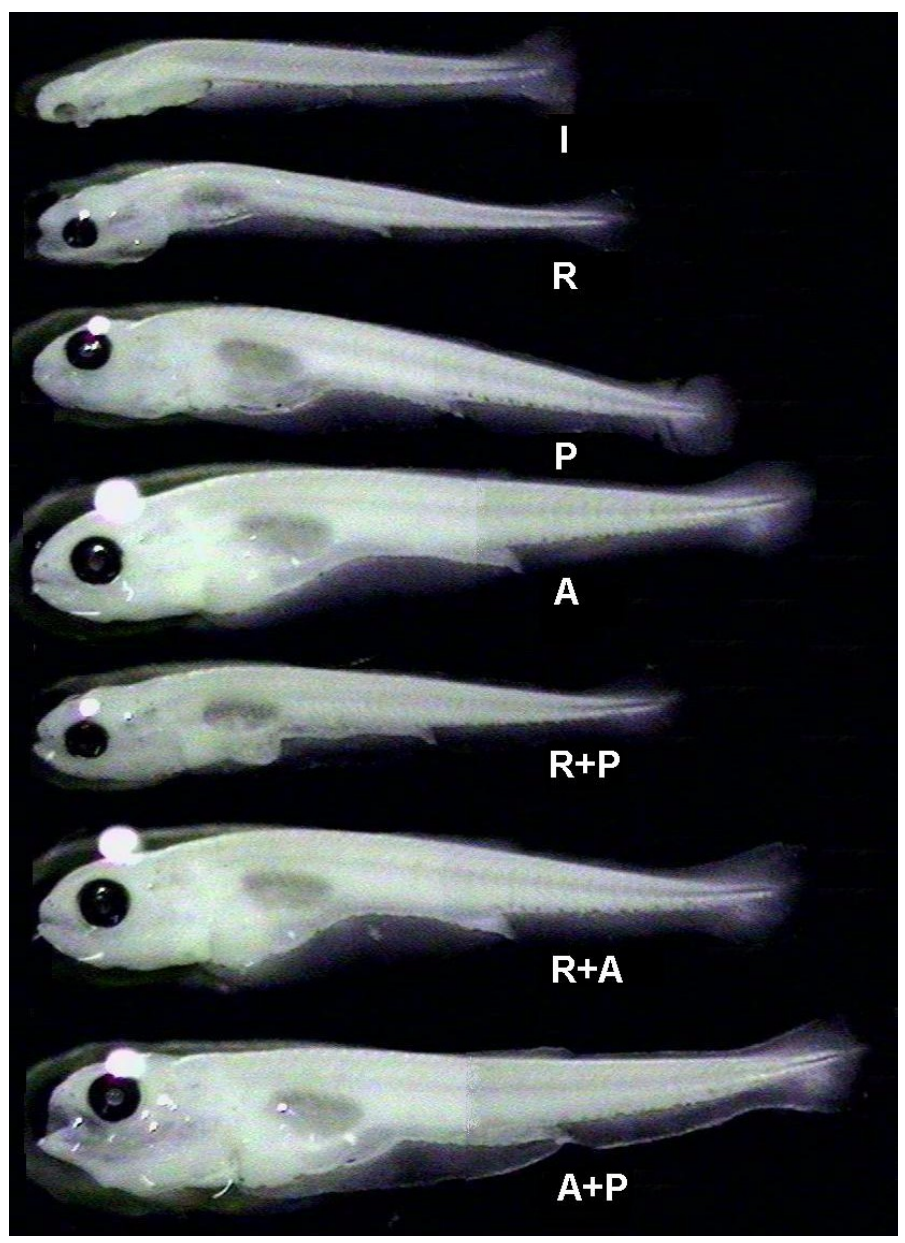

FIGURA 3 - Larvas de pacu, em que é mostrada a diferença de tamanho das larvas no início do experimento, com 2 dias de vida (I) e ao final, com 10 dias, em cada tratamento $\mathrm{R}=$ ração, $\mathrm{P}=$ plâncton, $\mathrm{A}=$ artêmia, $\mathrm{R}+\mathrm{P}=\mathrm{ra}-$ ção+plâncton, $\mathrm{R}+\mathrm{A}=$ ração+artêmia e $\mathrm{A}+\mathrm{P}=$ artêmia+plâncton.

Em relação aos tratamentos em que foram utilizados dois itens alimentares $(\mathrm{R}+\mathrm{P}, \mathrm{R}+\mathrm{A}$ e $\mathrm{A}+\mathrm{P})$, deve ser lembrado que cada item foi reduzido à metade da quantidade utilizada nos tratamentos em que o alimento foi fornecido isoladamente ( $\mathrm{R}, \mathrm{P}$ e $\mathrm{A})$. $\mathrm{O}$ tratamento composto por artêmia + plâncton foi o que proporcionou melhor comprimento total e peso, seguido pelo tratamento composto por ração + artêmia, e o pior desempenho foi obtido com o tratamento com ração + plâncton (Figuras 1 e 2).

O resultado do tratamento com artêmia + plâncton foi superior aos resultados obtidos com artêmia e plâncton fornecidos isoladamente, mostrando que a combinação desses alimentos foi eficiente, além de reduzir os custos com alimentação.
No decorrer do experimento, algumas características importantes referentes ao comportamento e desenvolvimento das larvas foram observadas. Essas observações têm importantes aplicações práticas, descritas a seguir.

No início do experimento, com dois dias de vida, as larvas não nadavam continuamente, ficando paradas quase todo o tempo no fundo das caixas e só nadavam ocasionalmente. Quando nadavam, era sem equilíbrio e quando paravam de nadar, afundavam. Logicamente, a ausência de nadadeiras desenvolvidas e a vesícula gasosa ainda não totalmente inflada impediam o nado constante e com estabilidade. Alguns autores também observaram que as larvas permanecem bastante tempo no fundo. Em larvas de dourado, essas se concentram no 
fundo das cubas no primeiro dia de vida, sendo esse comportamento reduzido a partir do segundo dia de vida (LUZ et al., 2000). Comportamento semelhante foi relatado por Santos e Godinho (1992), que estudaram o comportamento de larvas de 6 espécies de peixes sob condições experimentais.

Com o passar do tempo, e com seu desenvolvimento gradual, as larvas aumentam a frequiência do nado, até que, por volta do sexto dia de vida, elas não mais descansam no fundo e apresentam nado contínuo. Esse seria um bom momento para transferir as larvas para os viveiros escavados. De acordo com a prática usual, as larvas são transferidas com 2 ou 3 dias de vida, quando ainda não apresentam nado contínuo. Segundo observações nesse experimento, as larvas deveriam ser mantidas em laboratório, sob condições controladas e recebendo alimento, pelo menos até o sexto dia de vida.

\section{CONCLUSÕES}

De acordo com as condições deste experimento, conclui-se que:

a) A dieta artificial (ração) foi inadequada como primeiro e único alimento para larvas de pacu, devido ao baixo crescimento;

b) Isoladamente, a utilização de náuplios de artêmia como primeiro alimento para larvas de pacu é indicada;

c) A associação de náuplios de artêmia com plâncton foi o tratamento que proporcionou melhor desempenho, além de apresentar menor custo que o tratamento constituído apenas por artêmia; d) As larvas devem permanecer em laboratório por um período de 6 dias após a eclosão, recebendo alimento do terceiro ao sexto dia.

\section{REFERÊNCIAS BIBLIOGRÁFICAS}

FERREIRA, D. N. Sistema de análise estatística para dados balanceados. Lavras: UFLA/DEX/SISVAR, 1998.

JOMORI, R. K. Estudos sobre a alimentação de larvas de pacu, Piaractus mesopotamicus (Holmberg, 1887) com náuplios de artêmia e a sua substituição por dieta artificial. Jaboticabal: UNESP, $1999.70 \mathrm{p}$.

LUZ, R. K.; FERREIRA, A. A.; REYNALTE, D. A. T.; MAFFEZZOLLI, G.; ZANIBONI FILHO, E. Larvicultura de dourado (Salminus maxillosus, Valenciennes, 1849), nos primeiros dias de vida. In: SIMPÓSIO BRASILEIRO DE AQUICULTURA, 11., 2000, Florianólpolis. Anais... Florianópolis: [s.n.], 2000. CD-ROM.

SANTOS, J. E.; GODINHO, H. P. Comportamento de larvas de 6 espécies de peixes sob condições experimentais de fotoperíodo. In: ENCONTRO ANUAL DE AQUICULTURA DE MINAS GERAIS, 10., 1992, Belo Horizonte. Anais... Belo Horizonte: ABRAq, 1992. p. $75-77$.

WOYNAROVICH, E.; HORVATH, L. A propagação artificial de peixes de águas tropicais: manual de extensão. Brasília, DF: FAO/CODEVASP/CNPq, 1983. $220 \mathrm{p}$. 